\title{
Raíces Históricas de la Industria Colombiana
}

\section{Henry Rodríguez Sossa Coordinador de Investigaciones, Facultad de Administración Universidad Externado de Colombia}

Apenas terminadas las guerras de Independencia, comenzaron en Bogotá los intentos para lograr la Industrialización. Los historiadores coinciden en señalar que en el período 1830-1845 se desató en el centro del pais un marcado interés por tal objetivo, que incluso ha sido llamado "primera industrialización" quizá no tanto por sus logros como por la magnitud del empeño ${ }^{1}$

Después de algunos devaneos iniciales, donde se destaca con nombre propio el de la Ferrería de Pacho ${ }^{2}$ cuyo montaje comenzado en 1824 , obtiene el privilegio respectivo con decreto del 21 de agosto de 1827 otorgado a la compañía EGEA, DASTE Y CIA, empresa que inició operaciones en 1835-y especialmente a partir de 1830 , la élite neogranadina tomó en sus manos la responsabilidad de la industrialización, así se fundaron:

1834 Fábrica de loza reestructurada en 1837 que publicaba sus avisos en la Gaceta Nueva Granada.Contaba con dos molinos de tracción animal para la trituración de materiales, tres estufas, siete hornos, dos tipo Slip para elaborar pastas, tenía 60 operarios, cuatro técnicos extranjeros y un administrador. La "Sociedad Industrial Bogotana" que era su propietaria estaba constituída por Antonio Leyva Alvarez, Nicolás Leyva y Luis Montoya. Según la Gaceta, la fábrica pensaba exportar loza fina y porcelana. Es factible que sea la misma que tomará el nombre de FAENZA al comenzar el siglo $\mathrm{XX}^{3}$.

1836 Fábrica de Tejidos. Esta fábrica en 1839 contaba con 15 telares y maquinaria para hilados, utilizaba como fuerza hidráulica el Río San Francisco. Para 1852 aparece cerrada.
Fábrica de papel de Benedicto Domínguez. Según el registro de la Notaría Primera, contaba con edificio propio, máquinas para despedazar trapo y reducirlo a papilla, agua suficiente y posibilidades de montaje de otra rueda hidráulica.

También en los registros de la notaría primera aparece la Sociedad "Bordone, Saint Amand Bostaine y Compañía Sociedad Industrial" con objeto de montar un aserrío y negociar en ganadería. La historia del aserrío no nos es conocida ${ }^{4}$

1837 Fábrica de vidrios y cristales. Alcanzó a poner en venta sus productos pero tropezó con problemas de mano de obra, materias primas y mercados, dos años después había sido cerrada.

Fábrica de sombreros. Montaje iniciado por John Stewart, quien se quejó reiteradamente por problemas de disciplina de la mano de obra bogotana. Sin embargo en 1840 Pithon Ydobiesk, anuncia en la Gaceta nuevos tipos de sombreros, calidades y descuentos según el material empleado en su fabricación'.

1841 Fábrica de Paños de Filtro. Funcionó un año, al cabo del cual quiebra parece ser por dificultades financieras.

Desde los tiempos coloniales existía una fábrica de pólvora establecida por el Virrey Messia de la Cerda y en el período de la Gran Colombia se registra una fábrica de cerveza creada por el alemán Mayer, asesinado en 1831, la fábrica fue retomada por el inglés Cantrell y finalmente comprada por la sociedad "Martinez y Galinee" en 1834. Otro inglés Tomás Thompson anuncia los 
productos de su fábrica de cerveza y hasta 1840 se registraron dos tipografias y tres imprentas.

No es propiamente hablando un Fortín Industrial, pero el impulso les alcanzó para superar la guerra de 1840 y para participar en las ferias industriales que para resarcirlas impulsó la administración Herrán. Estas ferias se realizaron de 1841 a 1844 cada año, convocadas por la administración central se invitaba a industriales, artesanos y agricultores. El primer año generó gran expectativa pero posteriormente primó la tendencia de otorgar los mejores premios a las obras viruosas y de agrado antes que a las prácticas y útiles, tendencia que, por demás, se acompasaba muy bien con las tradicionales costumbres santafereñas $^{6}$

Desde el punto de vista de su estructura, se trataba de fábricas no de talleres artesanales, es decir, existía una separación rigurosa entre el propietario de los medios de producción (máquinas y capital) y los poseedores de la fuerza de trabajo, contratados por un salario. Por lo que conocemos, la forma típica de constitución de estas fábricas era la sociedad anónima pero con un reducido número de acciones de alto valor y por supuesto muy pocos accionistas. Esta situación es plenamente congruente con la mil veces descrita pobreza franciscana del país, la escasez monetaria y la alta concentración del ingreso. Frank Safford reseñó como miembros de la élite neogranadina que se vinculó a la industria a Ignacio Gutiérrez Vergara, Rafael y José María Alvarez, el coronel Joaquín Acosta, José María y Angel Chávez, destacando cómo "todos" poseían parentesco directo con funcionarios de la colonia y/o los grandes hacendados del período'.

Rufino Cuervo presentó en 1838 a nombre de los industriales, un proyecto de ley para concertaje de jóvenes, proyecto que aparece suscrito por José María Saíz, Marino Calvo, José María Calvo, Gregorio Gutiérrez, Miguel Saturnino Uribe, Rafael Alvarez, Joaquín Escobar, Félix Castro, José Joaquín Castro, Ignacio Morales, Benedicto Domínguez, Ramón Tamayo, José Ignacio París, Manuel Laverde, Santiago Grajales, José María Saravia, Francisco Sandino, Tiburcio Pieschacón, Cayetano Navarro, José María Saravia, Luis María Montoya y Judas Tadeo Landinez.
Estos 22 empresarios firmantes del proyecto fueron senadores o representantes de la República, evidenciando su vinculación abierta y directa en la política partidista y más exactamente en la labor que Marco Palacios denominó "Inventar la Nación".

Parece ser que la definición de las formas políticas: Estado, partidos políticos, relaciones Estado-sociedad civil, Iglesia-Estado, etc., produjo una ampliación y fortalecimiento del ámbito de la jurisprudencia a la par que la polarización del debate ideológico. Los dos fenómenos, concomitantes, condujeron a un "descuido" por los asuntos prácticos, útiles, industriales. Paradójicamente, tanto el fortalecimiento de los valores cristianos más tradicionales, como el discurso jurídico de la igualdad y la libertad, impidió una mayor difusión de los criterios positivos, seculares y pragmáticos en desmedro de una ética favorable al desarrollo de la industria. No ocurre necesariamente lo mismo con el sentido de enriquecimiento que se realiz6, en este caso, en la esfera del comercio.

La distinción es, a nuestros ojos muy importante, ya Max Weber lo había puesto de presente: lo definitivo en el surgimiento del Capitalismo no es el afán de lucro sino la forma, la manera de obtenerlo y esta manera es la que no está presente en nuestro caso.

La nueva ética industrial tampoco estaba presente en la mano de obra disponible como lo atestiguan numerosos testimonios de técnicos y empresarios y especialmente las leyes sobre vagancia de 1826 y 1836 de la naciente república.

Desde otro punto de vista y con respecto a las condiciones estructurales de la economia, se ha documentado suficientemente el problema de las materias primas y la gran dificultad técnica, solucionada así sea pirricamente a través de los técnicos extranjeros. Interesa observar que a pesar del gigantismo de planta señalada por Safford y las dificultades técnicas de implementación, las fábricas lograron producir, para encontrarse conque en mayor o menor medida todos habían sobrevaluado el mercado, es decir no vendían sus productos.

La razón profunda de esta limitación, debe buscarse más que en los posibles errores de administración, en el tipo de sociedad en la cual 
habían iniciado operaciones, el ahora llamado contexto. En suma, fue esta sociedad bogotana de medio siglo, pueblerina, atrasada y pachorra la que entregó tales limitaciones. Una sociedad que indudablemente NO ERA MERCANTIL y esta es una de las condiciones naturales para el desarrollo del capitalismo ${ }^{8}$. Es el mismo fenómeno que permitió el auge, desarrollo y caída de Judas Tadeo Landinez cuya compañía de Giro y Descuento se convirtió en el primero y único banco no sólo de Santa Fe sino de todo el interior del país, mientras el presupuesto nacional ascendía a dos millones de pesos, los activos de Landinez llegaban a la mitad y sus obligaciones le igualaban, Landinez resultó, gracias a sus maniobras especulativas, propietario directo de las fábricas de tejidos de algodón, de loza y de la Ferrería de Pacho, su quiebra fue un golpe que la débil economía santafereña no pudo soportar y que espantaría de la mente de los bogotanos cualquier iniciativa bancaria por casi 30 años. Los efectos pues, fueron devastadores; don José Manuel Restrepo lo consignaría en su Diario Político y Militar. "Más de 200 familias quedaron reducidas a la miseria por las maniobras atrevidas y avezadas de Landinez, cuya memoria será en Bogotá de funesta recordación. Sus deudas alcanzaron dos millones de pesos y sus propiedades apenas valen $500.000 " 9$.

En una economía atrasada, precapitalista, sin la composición social que posibilitara alterarla, con una élite que aunque deseo hacer industria distaba demasiado de la burguesía europea que creó el capitalismo y sin la voluntad política expresada en políticas estatales adecuadas, el porvenir industrial desapareció del horizonte bogotano. En 1846, el secretario de Hacienda don Florentino González expresa nítidamente este sentimiento:

"En un país rico en minas y productos agrícolas, que puede alimentar un cuantioso y lucrativo comercio de exportación, las leyes no deben favorecer el desarrollo de industrias que distraen a los habitantes de las ocupaciones extractivas y agrícolas que les permiten obtener la mayor ventaja.

Debemos ofrecerle a Europa, materias primas y abrir nuestros puertos a la manufactura para facilitar el comercio y las ventajas que él trae, para suministrarle al consumidor a precios bajos los productos de la industria manufacturera" 10 .
Como está plenamente establecido, no fue únicamente entre los aladides del Partido Liberal que se impuso este criterio. Don Mariano Ospina, a propósito del interés del dinero declaraba en 1834 su fe inquebrantable en la libertad de industria, como se estilaba decir a propósito del libre cambio, libertad que destaca como pilar del desarrollo en su célebre alegato de "la civilización" de 1849, una de las mayores exposiciones de la doctrina del Partido Conservador ${ }^{11}$. Así pues, a finales de los cincuentas, uno de los más lúcidos ideólogos de nuestro siglo XIX, don Salvador Camacho Roldán hace el balance:

"No hay que engañarse, mientras Bogotá no tenga vías de comunicación que la pongan en contacto con poblaciones consumidoras del Norte y del Sur, ningún progreso industrial podrá acometerse con buen suceso. Hay en esta ciudad una fábrica de cristales montada hace más de 30 años y no se ha pensado en establecer otra, la fábrica montada en 1838 tuvo que convertirse dos años más tarde, por falta de salidas, en hospital de virulentos; por lo que la malicia bogotana cambió el nombre por el de fábrica de virulentos; la fábrica de papel se convirtió en molino de trigo, la de tejidos de algodón se cerró, con inauditos esfuerzos de inteligencia, perseverancia y energía $\sin$ reconocerse fortuna y reputación a sus empresarios, la fábrica de tejidos de lana apenas repartía utilidades pequeñas a los señores Sánchez, Ponce y Cía., el laboriosísimo señor Eustasio Santamaría ha suspendido la fabricación de jabones y bujías...

Prototipo del comerciante, don Salvador expone fielmente el nuevo rumbo que tomó el país y la secreta alegría que le causaba:

"A cambio de abundante surtido de mencancías extranjeras y plazos verdaderamente liberales que se conceden en esta ciudad" 12 .

Bogotá abandona casi toda perspectiva industrial a la mitad del siglo y su élite dedica sus esfuerzos al comercio. Frustradas las aspiraciones de industriales e incapaces de plantearse el asunto como un reto ineludible de la modemidad, emprenden el camino especulativo comercial como forma de acumulación.

Una cuidadosa revisión de los registros de la Notaría Primera y Segunda de Bogotá para el período 1850-1875 arrojó los siguientes resultados: ${ }^{13}$

De 121 empresas constituídas apenas 13, es 
Esta forma de concebir el desarrollo de una nación y sus vaivenes se refleja en el auge y montaje ya resefiado de sociedades comerciales y especiaimente, en las relativamente numerosas que tuvieron como objeto la explotación y tratamiento de los frutos de exportación a saber las quinas, el añil y el tabaco.

Desde el punto de vista industrial cada vez se hace más notoria la crisis. En 1868 Samuel Sayer instala la primera máquina de vapor con destino a un molino de trigo. La fabricación de harina por lo que sabemos, era un buen negocio: existian tres molinos movidos por fuerza hidráulica que monopolizaban al mercado, si se superaban las contingencias de la fuerza hidráulica se podría incrementar la producción y bajar los costos, de este razonamiento partio Sayer.

Según cuentan en la reciente y lujosa "Historia de Bogotá"15, los propietarios de los molinos hidráulicos se apresuraron a negociar con Saver el molino, quedándose éste con la máquina de vapor. Acto seguido ante alzas en la materia prima y de nuevo dueños absolutos del mercado, suspendieron la elaboración del pan de a cuarto, el más barato, y eliminaron el vendaje acostumbrado. La reacción de los bogotanos no se hizo esperar, se conoce como el "Motín del pan".

Don Santiago Pérez, presidente en ese entonces de la República manifestó ante los bogotanos que le estaba vedado intervenir en el conflicto para inclinar la balanza en cualquiera de los sentidos puesto que la constitución de la República consagraba la más irrestricta libertad de industria. A continuación expresó que si era verdad que los panaderos estaban cometiendo abusos en los precios de este artículo de primera necesidad, él confiaba en que, ante la protesta popular, los fabricantes de pan entraran en diálogo con las gentes para llegar a una feliz conciliación entre sus intereses y los de sus consumidores.

Concluyó su alocución pidiendo a los manifestantes cordura y sosiego en su conducta y sus actividades, se despidió afablemente de la muchedumbre y se retiró del balcón (Sic).

¡Una excelente muestra del discurso librecambista! Los bogotanos, por supuesto, no siguieron las orientaciones dadas por el ciudadano presidente y transformaron el motín en asonada. Más ilustrativo aún resulta destacar que la élite bogotana publicó un desagravio dirigido a don Joaquín Sarmiento, a la sazón presidente del Banco de Bogotá y propietario del mayor molino implicado en el problema "Los Alisos".

La lista de los firmantes confirma el patrón de actividades que ya se había establecido:

Santiago Pérez Vicente, Arzobispo de Bogotá, Roberto Bunsh, Eustargio Salgar, Aquileo Parra, Silvestre Samper, Miguel Samper. Jon María Samper, Salvador Camacho Roldán, Lorenzo Lleras, Víctor Mallarino, José M. Marroquín, Carlos Holguín, Jorge Holguín, José María Quijano W., Rafael Pombo, José María Saravia, Ramón del Corral, Florentino Vesga; Evaristo de la Torre, Rafael Portocarreño, Wenceslao Pizano, Pedro Dordelly, Félix M. Pardo Roche, Nepomuceno Santamaría T.M. Quijano Otero, Vicente Lafaurie, Eustacio de la Torre, Carlos B. Bunsh, Emiliano Restrepo, José A. Obregón, Tomás E. Abello, Mariano Tasco, Manuel Ponce de León y Jacobo Sánchez ${ }^{16}$

En 1874, también se cierra la fábrica que pretendió hacer ácido sulfúrico y sólo alguna fábrica de cigarros (Koppel \& Shools, 1874) y la fábrica de cerillas Rey-Borda creada en 1870 constituían "La Industria" Bogotana. La situación se manifiesta claramente en el siguiente hecho: Para la época, en prácticamente toda Cundinamarca sólo se levantaba una chimenea.

Sin embargo, Bogotá crecía: Si en 1793 el censo declaraba que tenía 17.725 habitantes, suma que en 1801 se redondea en 21.349 para 1843 el censo indica 40.086 habitantes, cantidad que se duplica en 1898. Esto implicaba necesariamente un incremento del mercado, sobre todo si encontraba cierta acumulación y esto es lo que atestigua el resurgir del sector bancario.

Luego de la quiebra de Judas Tadeo Landinez funcionó en Bogotá de 1846 a 1863 la caja de ahorros, con pagos del $1 \%$ el $2 \%$ e intereses del $10 \%$ anual, según las crónicas bueno para la época.

En 1864 se fundó el Banco de Londres, México, Sudamérica duró un año, no quiso aceptar socios nacionales y se transformó en una agencia, pero justamente en 1870 existían los capitales suficientes como para sostener este tipo de instituciones y en 1871 se fundó el Banco de Bogotá; cuatro años más tarde se establece la competen- 
cia, el Banco de Colombia y en 1875 entra en funcionamiento la Compañía Colombiana de Seguros, creada por iniciativa del santandereano Pedro Navas Azuero el af́o anterior; para 1881 tendremos 8 Bancos en la capital y 42 a nivel nacional ${ }^{17}$. Difícilmente encontramos un mejor indicativo de los resultados de la especulación comercial, por esto no es nada extrafio que cuando Núnez impulse en 1882 la creación del Banco Nacional se desate una de las pugnas más agudas que lanza a la oposición a toda la burguesía comercial.

En fin es este crecimiento vegetativo de la economía, sumado según parece, a la nueva percepción de los asuntos industriales inaugurada por el primer gobiemo de Núñez la que dio vida a cierta recuperación industrial bogotana a fines del siglo, recuperación donde ya aparecen los capitales formados en la esfera comercial y donde la capacidad técnica resulta cada vez más definitiva:

1888 En Albán se inauguró una tenería moderna, que rápidamente se extendió a fábrica de calzado, correos de transmisión y otros artículos de cuero. Poveda Ramos reseña que subsistió hasta 1905 de manera eficiente.

1882 Indalecio Uribe, antioqueño, se traslada a Bogotá instalando una fábrica de tejidos, con telares de su propio diseño con base en la cual satisfacía la demanda bogotana. Superada la nueva guerra y la fuerte crisis internacional podemos entrever el resurgimiento industrial.

1888 Se organiza la "Sociedad Industrial de Bogotá" con el propósito de revivir la fábrica de Acido Sulfúrico. Dos años más tarde vuelve a cerrarse; aún no existe la suficiente demanda. Este mismo año se cierra la fábrica de tejidos de lana después de 30 años de existencia. La razón es la incapacidad técnica de superar la pérdida de su fuente de energía: El Río San Francisco.

1889 Se funda la fábrica de Chocolates "La Equitativa" que en 1905 se fusiona con la fábrica de chocolates "Chávez" fundada en 1877 por Enrique Chávez con máquina movida a vapor. La nueva fábrica durará hasta nuestros días.

Leo S. Kopp funda Bavaria, la primera cervecería en escala apreciable montada en el país, con equipos y técnica moderna. Desde el primer momento tiene perdurables efectos: incentivó la producción de cebada en la sabana con el fin de garantizarse la materia prima, dio nacimiento a la industria del hielo y fue mercado de la industria del vidrio.

1892 Se fundan dos fábricas de pasta "El Gallo" y el "Papagayo" su fusión existe hoy en día, se trata de pastas "Doria".

1897 Se crea "Fenicia" fábrica de envases de vidrio, creada para satisfacer el mercado de Bavaria su éxito fue inmediato.

Fábrica de Ladrillos de Ana Calvo de Philipps, importante por la innovación en técnicas y equipos que posibilitaron su subsistencia.

Poveda Ramos y Luis Ospina Vásquez reseñan además dos fábricas de vidrio, una de las cuales subsistió hasta bien entrado el siglo.

Este es el panorama que encontró la guerra de los mil días y el nuevo siglo: fábricas con una demanda intema firme pero pequeña, productores de bienes de consumo masivo, que respondían a necesidades más bien inmediatas de la población aunque por depender fundamentalmente de materias primas nacionales y poseer una capacidad técnica adecuada podían subsistir y lo hicieron.

Digamos finalmente que aún está lejos el momento en que Bogotá deje de ser una aldea y se transforme en el centro financiero e industrial que hoy conocemos.

\section{NOTAS}

1 El nombre fue propuesto por Anita Weis en "Anleceden. tes del Desarrollo Industrial Colombiano" Documentos No. 20 Sociología UN. Bogotá 1980.

2 Ver el excelente estudio de Tatiana Machler "La Ferre. ría de Pacho" tesis Facultad de Economía UN. Bogotá 1985.

3 La informaciṓn historiográfica está más o menos referenciada en los textos clásicos de: Luis Ospina Vásquez "Industria y Protección en Colombia 1810.1930" Ed. FAES. Medellín 1979.

Gabriel Poveda Ramos "Historia de la Industria" Revista Trimestral No. 11 ANDI Medellín. 1970.

Cuando se cree que es nueva la información se cita la fuente. 
4 Notarin Primere. Tomo XV Folios 207-210-211

Escribano Jose Lucio de Elorge A.M.N. Bogotá.

5 Gaceta de la Nueva Granada. Bogotá. Dorningo 30 de Octubre de 1836 No. 266.

6 Sobre las ferias industriales ver la tesis de José Rubén Parrado H. Industrial. Bogotí, 1825-1850 Facultad de Administración de Empresas. Universidad Extemado de Colombia. Bogoth, 1985.

7 Frank Safford "Empresarios nacionales y extranjeros en Colombia durante el Siglo XIX" en Anuacio Colombiano de historia social de la cultura. No. 651, Vol, XXV, Mayo 1975. U.Nacionsal, Bogotá.

8 Sobre algunos aspectos de la ética empresarial en la época ver nuestro trabajo "La Etica de los primeros industriales bogolanos". Ponencia presentada al "VII Encuentro Nacional de Investigadores en Administración". Bogoun 1988.

9 Citado por Mario Arango Jaramillo "Judas Tadeo Landi. nez y la primera Bancarrola Colombiana"
10 Jaime Duarne French, Florentino Gonzflez "Razbin yuin razón de una lucha polática" Carlos Valencis Editores, Bogota 1982.

11 Ver Mariano Ospina Rodríguez "Escrilos sobre Econo. mía y Politica" U.N. Dirección de Divulgación Cultural. Bogota 1969.

12 Salvador Camacho Roldán en "Misceld́nea". Bogotá 1958. Fondo Anseimo Pineda No. 20. Biblioteca Nacional, Bogotá.

13 Los registros notariales están transcritos en la tésis de Oscar Noe Pachón "La Industria Bogotana 1850 - 1875" Facultad de Administración de Empresas. Universidad Externado de Colombia. Bogotá 1985.

14 Por demás ha sido explicada y justificada por los historiadores contemporáneos como Marco Palacios, Jorge O. Melo y sobre todo José Antonio Ocampo.

15 Fundación Misión Colombia "Historia de Bogotá" Tomos 3. Ed. Benjamín Villegas \& Asociados. Bogotá 1988.

16 lbidem. Pag. 209.

17 Un resumen de la activid bancaria en cualquiera de los textos citados. 